\title{
Obituary
}

\section{Leon J. Thal, MD}

June 17, 1944 - February 3, 2007

On Saturday, February $3^{\text {rd }}$, 2007, Leon J. Thal, MD died in a tragic accident while flying his single engine airplane from San Diego to Borrego Springs, California. He is survived by his life-long comrade and wife, Donna Thal, PhD. The Alzheimer's disease (AD) research community deeply laments the passing of a universally loved and admired leader of the field. Personally, my grief is over the loss of a very kind, gentle and generous friend. Through the years, Leon had become a constant source of inspiration and the brother I never had.

\footnotetext{
“... Come hither, all you good people, Sit down and listen well

A wondering bard from distant parts A wondrous tale will I tell.

We all are guests in this mortal world

Since the day we get our birth.

We come and go, each in his turn,

To and from this fleeting earth.

We pass away, and only our deeds

Good or bad, live in fame,

So blessed be he who leaves the world

As pure a man as he came."

From - 'The Capture of Fort Temuk' by Hovannes Toumanian, 1903.

$$
* * *
$$

Leon Thal's entire career was devoted to the study of aging and dementia. Over nearly four decades, he pursued cures for dementia with unwavering commitment and passion. He was one of the world's leading authorities in the development of new therapeutics for AD. In the annals of dementia research he will be remembered as one of only a handful of scholars that have had a
}

significant impact on the processes of 'drug discovery' and 'therapy development.' ${ }^{1}$

Leon was a founder and a renaissance scholar, a modern-day Thomas Jefferson, with a diverse range of personal and intellectual interests. Although he had a singular passion for discovering a cure for dementia, he excelled in many domains of science and was extremely well-informed in different areas ranging from horticulture, natural history, mechanics, flying, and backpacking. The spectrum of his major scientific work covered a remarkable body of research with more than 300 peer-reviewed papers; these covered a broad range of basic and clinical questions concerning 1) the behavioral/clinical, biochemical, genetics and neuropathologic correlates of dementia; 2) clinical trials in Alzheimer's disease; 3) Biomarkers in Alzheimer's disease; and 4) discovery of interventions to enhance neuronal function and regeneration. The versatility of his scholarly pursuits is illustrated by the:

- Biochemical studies that demonstrated the presence of altered p59Fyn kinase expression in $A D$ and a persistent decrease in monoamine oxidase $\mathrm{A}$ $\&$ B. These findings help to define the biochemical alterations underlying the pathology of $\mathrm{AD}$ dementia.

- Clinicopathological studies with Dr. Tiraboschi and Corey-Bloom, which demonstrated that a) AD can occur even in the absence of neurofibrillary tangles; b) neuritic plaques are associated with the earliest symptoms of $\mathrm{AD}$; and c) the presence of two apolipoprotein E4 alleles is an important determinant of both neuritic plaque and neurofibrillary tangles accumulation.

- Genetic studies demonstrated an alteration in the $\alpha$-1-antichymotrypsin gene promotor region

\footnotetext{
${ }^{1}$ NOTE: Leon was a unifier and a bridge builder, and, in the spirit of his memory as a symbolic gesture, this obituary will be co-published in Alzheimer's \& Dementia: The Journal of the Alzheimer's Association, and the Journal of Alzheimer's Disease, journals that he loved and supported, to join forces in an effort to preserve his memory as a leader in our field.
} 
in $\mathrm{AD}$ and the association of late onset $\mathrm{AD}$ with genetic variability in the gene encoding glyceraldehyde-3-phosphate dehydrogenase (GAPD). These studies currently help to define susceptibility genes that play a role in the development of late onset AD.

- Neuropsychological studies of brain-behavioral relations demonstrated that: a) the relationship between age and memory functioning and executive functioning was modified by apolipoprotein E4 genotype; b) individuals with Lewy body dementia retain a better ability to consolidate information than do patients with $\mathrm{AD}$; and c) individuals with motor disorders and AD early in the course of their illness were more likely to develop psychosis in subsequent years. These three studies helped to characterize the neuropsychological phenotype of patients with AD and Lewy body dementia.

- In collaboration with Dr. Fred Gage, he studied strategies to manipulate cholinergic system with a neurotrophic factor aimed at enhancing neuronal function and regeneration.

Leon's primary and life-long interest in the discovery of treatments for dementia began in mid-1970s with studies using choline, lecithin and other precursors of acetylcholine to test the 'cholinergic hypothesis of AD' in animal models as well as studies with humans. In 1981, he published the finding that choline chloride failed to improve cognition in patients with $\mathrm{AD}$. This failure, however, motivated him to explore alternative strategies to treat the cholinergic deficit of AD using other compounds and routes of administration. Indeed, his perseverance paid off with when he discovered some of the first evidence that memory could be enhanced in AD patients with cholinesterase inhibition. The culmination of his intense research efforts was the approval of Tacrine [a cholinesterase inhibitor] by the Food and Drug Administration (FDA) as the first drug for the treatment of AD.

In 1987, in collaboration with Kenneth Davis, MD, Elkan Gamzu, Ph.D, the National Institute on Aging (NIA), the FDA and others, Leon organized the first formal multi-site clinical trial for evaluating a cholinesterase inhibitor as a potential treatment for AD. This double-blind, placebo-controlled, multi-center study was a landmark that paved the way for the approval of other similar-acting compounds; it continues to serve as a general template for testing the efficacy of putative treatments for cognitive impairments. His work in this period demonstrated his unique organizational skills and established his unquestioned lead- ership in the field of testing and development of therapies for dementia. No single person in the history of dementia research has made a larger impact on the experimental therapeutics of AD than Leon Thal.

In the early 1990, at the completion of the NIAfunded clinical trial with THA [Tacrine], the need for building-up the national infrastructure and capabilities for clinical trials led the NIA to conceive the Alzheimer's Disease Cooperative Study (ADCS) and Instrument Development Program. The universallyacknowledged successes of this program was due largely to Leon's leadership in forging partnerships and the enormous reservoirs of trust he engendered among all collaborators and participating institutions. Since the inception of the ADCS Leon has been the guiding force behind the efforts to develop and validate new instruments for evaluating the course of $\mathrm{AD}$, as well as for ADCS's work in developing and evaluating interventions aimed at delaying the course of the disease.

In nearly two decades of operations under Leon's leadership, the ADCS studies had a profound impact on therapy development and clinical practices for patients with $\mathrm{AD}$. The program has enrolled more than, 4,500 subjects in clinical trials and instrumentvalidation studies at 90 sites in the United States and Canada. Under Leon's leadership, 19 separate clinical or instrument protocols have been initiated and 13 have been published. The high profile his work enjoys reflects the importance of his clinical research in carrying findings from the bench to evaluation in humans.

The ADCS spearheaded improvements in the definition of prodromal stages of the disease and the development of new study designs and instruments useful for more rigorous clinical trials. For example, ADCS played a key role in refining the concept of MCI, a state where individuals have a memory deficit but do not meet the clinical criteria for AD. Individuals with MCI develop dementia at the rate of $12-15 \%$ per annum. Based on this idea, the ADCS initiated a large intervention study to examine the effects of vitamin $\mathrm{E}$, an anti-oxidant, and Donepezil, a cholinesterase inhibitor, to prevent conversion from MCI to AD. The results of this three-year clinical trial, with a cohort of $769 \mathrm{MCI}$ subjects, demonstrated that Donepezil delayed the time to the conversion from MCI to AD by 12-18 months. Vitamin E had no effect. This study also showed that: 1) MCI can be distinguished from $A D$ and normal aging; 2) hippocampal volume loss is associated with memory but not with non-memory cognitive impairment in patients with MCI; 3) brain hippocampal volume predicts cognitive status in MCI; and 4) the pres- 
ence of the apolipoprotein E4 allele is associated with a significantly reduced hippocampal volume.

A partial list of the clinical trials conducted by the ADCS from 1991 to 2007 includes: Vitamin E and Selegiline 1992-1995; Instrument protocol (English and Spanish) 1993-1995; Haloperidol and trazodone for agitation 1994-1997; Prednisone as an anti-inflammatory 1995-1998; Conjugated equine estrogens 1995-1999; Melatonin for sleep disturbance 1997-2000; Vitamin E, Donepezil for mild cognitive impairment 1999-2004; MCI instrument development 1999-2004; Naproxen and rofecoxib as antiinflammatories 1999-2001; Valproate for agitation 2000-2002; Instruments for prevention trials 20022006; Cholesterol-lowering with simvastatin 20032007; Homocysteine reduction with B vitamins 20032007; Valproate for neuroprotection 2003-2009; and, Anti-oxidant, DHA 2007.

In September 2004 Leon Thal, in partnership with Michael Weiner, MD, assumed the leadership for yet another multi-site collaborative project; the Alzheimer's Disease Neuroimaging Initiative (ADNI) funded by NIA. The goal of this longitudinal observational study of individuals with no symptoms, MCI, or early AD was to improve the instruments and methods for detecting or measuring the progression of early dementia in clinical trials. The ultimate aim was (and still is) to develop a national infrastructure for standardizing and validating various specific biomarkers [including neuroimaging] for tracking changes in people at risk or early stages of the disease. Thereby we will improve the technology needed to detect disease-modifying effects of treatments earlier in the course of a clinical trial. Leon served as the director of the clinical core responsible for recruiting 800 subjects at 59 sites in the United States and Canada and for obtaining and managing clinical data derived from this initiative. (In this project, Michael Weiner at UCSF currently serves as the overall principal investigator.) This public/private initiative involves the participation and cooperation of two NIH institutes, eleven pharmaceutical companies, and two foundations.

Leon's most recent forward looking project on therapy development was the creation of the Lou Ruvo Brain Institute [LRBI] in Las Vegas, Nevada. The mission of the Institute is to accelerate the discovery of cures for memory disorders associated with neurodegenerative diseases such as Alzheimer's, Huntington's, Parkinson's, ALS and other brain dysfunctions. The aim of the LRBI is to foster the creation of worldwide cooperative research networks to: a) develop new de- tection technologies for early and accurate diagnosis of dementia and b) facilitate the development of treatments for various forms of memory impairments. Leon served as a senior advisor to this project and the Chair of the External Scientific Advisor Board. The idea of this institute was conceived by Larry Ruvo, a Las Vegas businessman and philanthropist, to honor his father's memory; Lou Ruvo had Alzheimer's disease and Leon was his physician. Today, this institute has to honor not only the memory of a beloved father with the disease, but also the physician who was so dedicated to finding a treatment and a cure.

$$
* * *
$$

Leon obtained his undergraduate training at Tufts University [BS - Magna cum laude, 1965]. He received his medical training at Downstate Medical Center [MD - Summa cum laude, 1969]. His postgraduate training included: Internship in Medicine at Kings County-State University Hospital, 19691970; Resident in Neurology, Albert Einstein College of Medicine, 1972-1975; Fellow, Interdepartmental Research Training Program Albert Einstein College of Medicine, 1975-1977; Visiting Scientist, Department of Pharmacology, Johns Hopkins University, with Solomon Snyder, MD, 1976.

His academic carrier began at the Albert Einstein College of Medicine Bronx, NY, as an Instructor in Neurology [1975-1977] rapidly advancing to the positions of Assistant Professor of Neurology [1977-1982], Associate Professor of Neurology [1982-1985]. In 1985 he moved to University of California San Diego, CA, [along with Drs. Robert Katzman and Robert Terry, his early mentors and later his collaborators] as an Associate Professor of Neurosciences [1985-1989]. At UCSD he was promoted to the rank of Professor of Neurosciences [1989-2007] and eventually to the Chair of the Department of Neurosciences [1993-2007]. Later still, he achieved the much-coveted position of Florence Riford Chair in Alzheimer's Disease [1994-2007].

He was a member of the American Academy of Neurology, the American Neurological Association, the International College of Geriatric Psychoneuropharmacology and the American Association for the Advancement of Science. He served on the editorial boards of numerous scientific journals including Alzheimer's \& Dementia: Journal of the Alzheimer's Association, Neurobiology of Aging, and, Journal of Alzheimer's Disease.

The awards and recognitions he received includes: Department of Neurosciences Senior Resident Teach- 
ing Award, 1987-1988; Department of Neurosciences Outstanding Teaching Award, 1989-1990; American Legion Dedicated Service Award, February, 1989; Consiglio Nazionale delle Richerche, Rome, Italy, 1990; Lifetime Science Awardee, Institute for Advanced Studies in Immunology; and Aging, Washington, DC, 1992; Department of Neurosciences, Clinical Teaching Award, 1993-1994; Florence Riford Chair in Alzheimer's Disease, 1994 - present; National Advisory Council on Aging of the National Institutes of Health, 2002-2005; Arthur Cherkin Memorial Award, 2003; Potamkin Prize for Research in Pick's, Alzheimer's and Related Diseases, 2004; Distinguished Scientist Award, International College of Geriatric Psychopharmacology, Pittsburgh, PA, 2005; the Alzheimer's Association's 'Zaven Khachaturian Award', 2006; and the Ronald Reagan Legacy Award, 2007.

Some of the significant committee or board appointments he had included: Chairman, FDA Peripheral and Central Nervous System Drugs Advisory Committee, 1989-1992; Head, AAN Geriatric Neurology Research Committee, 1989-1998; Director of Research Programs for Geriatrics Section, American Academy of Neurology, 1989-1998; FDA Advisor, Alzheimer Assessment Team, 1992-2007; World Federation of Neurology, Research Group on Dementia, 1996-2007; Scientific Advisory Board, San Diego Alzheimer's Association, 1997-2007; French Foundation Scientific Advisory Board, 1998-2007; Appointee, Independent Citizens Oversight Committee of the California Institute for Regenerative Medicine, 2004 - present; Alzheimer's Association Advisory Workgroup, December 20042007; Senior Scientific Advisor and Chair, External Scientific Advisory Board, The Lou Ruvo Brain Institute, 2005-2007; Associate Editor, Alzheimer's \& Dementia, 2006-2007; Alzheimer's Association
National Board, 2006-2007; Scientific Board, Research and Practice in Alzheimer's Disease and Other Dementias, 2007.

Leon Thal was a rare leader in a highly competitive field. He had a unique compass for finding a middle ground in any contentious scientific discourse or dispute. His life and academic career, like a diamond, had many brilliant facets: he was an outstanding physician, a keen teacher, a brilliant scientist, and a loyal friend. As a mentor to young investigators and as the trustee and sole administrator of an anonymous endowment to help new researchers, he affected the lives and careers of countless individuals within UCSD and around the country. Some the individuals he trained at USCD includes: Ronald Mandel, Donald Connor, Ad Dekker, Jerene Waite, Jurgen Winkler, Don Pizzo, Bruce Lasker, Regis Kwo, Douglas Galasko, Jody Bloom, John Olichney, Ron Ellis, Ron Ellis, Bill Samuel, Marwan Sabbagh, Ursula Hohl, Barbara Schoos, Gilbert Ho, Gang Tong, Annette Merdes, Chuang-Kuo Wu, Adam Fleisher, Roy Yaari, David Weisman, Abdullah Sherzai, and Michael Rafii.

Leon Thal's three decades of substantive contributions to the understanding, prevention, and treatment of $\mathrm{AD}$ and related disorders have resulted in new pathways for scientists to create a new paradigm for clinical practice and diagnosis. The Alzheimer cause mourns the loss of an essential and unforgettable leader.

Zaven S. Khachaturian, $\mathrm{PhD}$

President \& CEO

Lou Ruvo Brain Institute, USA

E-mail: zaven_khachaturian@kra.net 